\title{
Abdominal Pain and Fever Indicative Postpartum Ovarian Vein Thrombosis: A Case Report
}

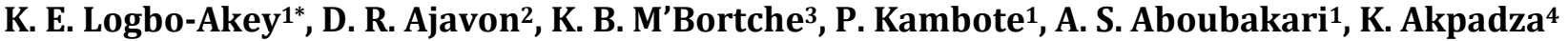 \\ ${ }^{1}$ Department of Gynecology and Obstetrics, CHU Kara, University of Kara, Kara, Togo \\ ${ }^{2}$ Gynecology and Obstetrics Department of CHR Tomdé, University of Kara, Kara, Togo \\ ${ }^{3}$ Clinic of the Togolese Association for Family Welfare, University of Lome, Lome, Togo \\ ${ }^{4}$ Department of Gynecology and Obstetrics, CHU Lome, University of Lome, Lome, Togo \\ Email: *edemattis@yahoo.fr
}

How to cite this paper: Logbo-Akey, K.E. Ajavon, D.R., M'Bortche, K.B., Kambote, P., Aboubakari, A.S. and Akpadza, K. (2021) Abdominal Pain and Fever Indicative Postpartum Ovarian Vein Thrombosis: A Case Report. Open Journal of Obstetrics and Gynecology, 11, 1378-1385.

https://doi.org/10.4236/ojog.2021.1110128

Received: September 12, 2021

Accepted: October 18, 2021

Published: October 21, 2021

Copyright (c) 2021 by author(s) and Scientific Research Publishing Inc. This work is licensed under the Creative Commons Attribution International License (CC BY 4.0).

http://creativecommons.org/licenses/by/4.0/

\begin{abstract}
Introduction: OVT is rare, with an incidence of $0.05 \%$ to $0.18 \%$ and occurring preferentially in postpartum period. Any delay in management may compromise the patient's vital prognosis. Observation: We report a case of a 34year-old patient, G2P3, who delivered twins by cesarean section at 39 weeks of amenorrhea. She presented abdominal pain and fever on the $4^{\text {th }}$ day of postpartum. Biological tests showed a biological inflammatory syndrome and a positive blood culture with gram-positive Staphylococcus. After 72 hours of antibiotics with no clinical or biological improvement, a CT scan revealed a $12 \mathrm{~mm}$ thrombus in the lumen of the right ovarian vein's connection to the inferior vena cava. Antibiotic treatment was continued, associated with an anticoagulant. The evolution was been favorable. Conclusion: Rare and serious pathology, the diagnosis is made using medical imaging. The outcome is generally favorable with antibiotic therapy and anticoagulant therapy.
\end{abstract}

\section{Keywords}

Abdominal Pain, Fever, Post Partum, Ovarian Vein Thrombosis

\section{Introduction}

Ovarian vein thrombosis (OVT) is a fairly rare and extremely serious pathology that can be life-threatening for the mother if the diagnosis is unrecognized. It occurs preferentially in the postpartum period, with a variable incidence of $0.05 \%$ to $0.18 \%$ of vaginal births, to $2 \%$ after cesarean section [1] [2] [3]. Discovered for 
the first time in 1956 by Austin [4], the pathology predominates on the right in $80 \%$ to $90 \%$ of cases [5]. By its painful abdominal manifestations and fever, it can be confused to other painful and feverish abdominal pathologies that lead to misdiagnosis. Through progress advances in medical imaging, the diagnosis is made early allowing adequate treatment and avoiding major complications, such as pulmonary embolism and sepsis [6]. We always think about it in the postpartum period whenever a woman has abdominal pain with fever, especially by caesarean section.

We report our management of postpartum ovarian vein thrombosis through this case report.

\section{Case Presentation}

We report the case of a 34-year-old patient, G2P3, without particular history, not-smoker, who delivered at 39 amenorrhea weeks by cesarean section, twins who were in transverse position. The pregnancy was obtained by in vitro fertilization. Four days after the caesarean, she has felt abdominal and pelvic pain in the right iliac fossa extending up into the right flank, accompanied by breath difficulty and hyperthermia. The abdomen was painful on palpation. The vaginal examination was painful overall, but did not find anything. The biological assessment noted an hyper leukocytosis at 21,000 white blood cells with a progressive increase of C-reactive protein to 295 and thrombocytosis. The polymerase chain reaction test for COVID-19 and an urine bacteriological examination were negative. A vaginal swab and blood culture revealed gram-positive Staphylococcus infection. Antibiotic treatment was then instituted. In view of breathing difficulties, a pulmonary CT (Computed Tomography) angiography was performed but did not find any signs suggestive of pulmonary embolism. The persistence of abdominal pain and fever despite antibiotic therapy 72 hours later, prompted the performance of a pelvic thoraco-abdominal CT scan which showed a $12 \mathrm{~mm}$ thrombus of the right ovarian vein with inferior vena cava (IVC) extension (Figure 1 and Figure 2 showing the thrombus in the lumen of the IVC, Figure 3 showing the thrombus in the right ovarian vein, with extension into the IVC). The diagnosis of thrombosis of the right ovarian vein was then retained.

Antibiotic treatment was continued, associated with low molecular weight heparin. The evolution was favorable with the disappearance of abdominal pain and fever, and the normalization of biological infectious parameters. A pelvic abdominal CT scan confirms the good evolution with the disappearance of the thrombus IVC (Figure 4). The patient left the hospital seven days later with low molecular weight heparin for 3 months and antibiotics. She was seen in one month later. Clinical examination was normal as well as infectious biological parameters.

\section{Discussion}

\section{Epidemiology}

TVO is a rare, occurring preferentially in the postpartum period. Its incidence 


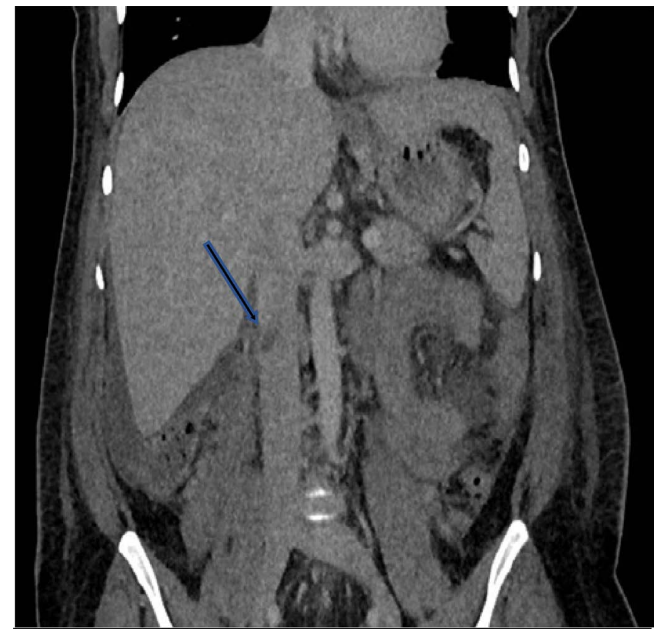

Figure 1. The arrow shows the thrombus in the inferior vena cava.

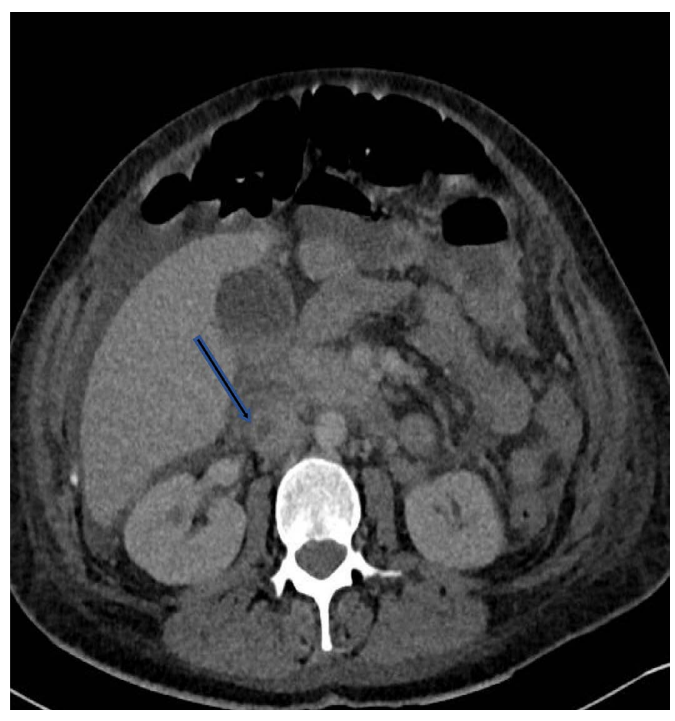

Figure 2. Axial view, the arrow shows the thrombus in the inferior vena cava.

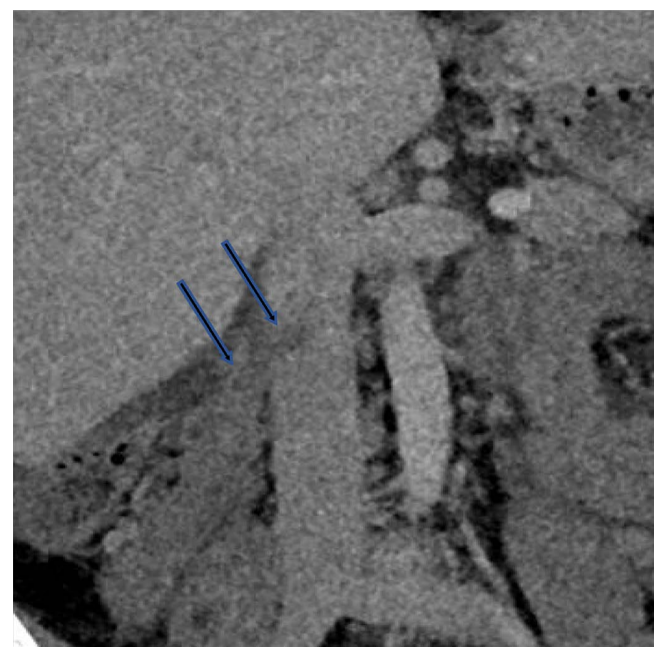

Figure 3. Arrows show thrombus in the right ovarian vein with extension into the inferior vena cava. 


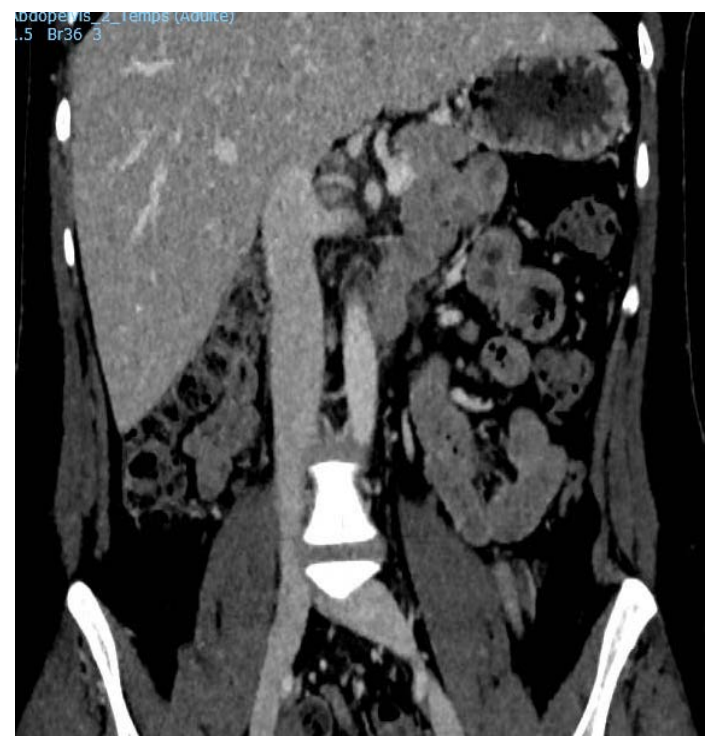

Figure 4. Coronal view, disappearance of the thrombus in the inferior vena cava.

varies from $0.05 \%$ to $0.18 \%$ of vaginal deliveries, or $1 / 2000$ to $1 / 600$ births. This incidence would be higher after a delivery by cesarean section. An incidence ranging from $0.42 \%$ to $2 \%$ after cesarean section has been reported in literature [3] [7]. This is a serious pathology with a mortality with lethality close to $5 \%$ even when diagnosed early [3] [6]. It occurs in patients between 30 and 40 years of age, most often in multiparous women [5] [7] as in our case reported.

\section{Etiopathogenesis}

The etiopathogenesis of OVT during pregnancy and postpartum results from Virchow triad which includes venous stasis, endothelial damage and hypercoagulability [8] [9]. The postpartum period is a special period of blood hypercoagulability, increasing the risk of thromboembolism. It may be increased by a genetic or acquired predisposition to venous or arterial thrombosis, in particular resistance to activated protein $\mathrm{C}$, deficiency in antithrombin III, protein C, protein $S$, hyperhomocyteinemia and antiphospholipid syndrome, mutation of the factor II or factor V Leiden [10].

Venous stasis is thought to be the result of IVC compression by gravid uterus, and venous dilation by hormonal imbibition during pregnancy [11]. In addition, involvement of the ovarian vein predominates on the right in $80 \%$ to $90 \%$ of cases [5]. This increased incidence of the right ovarian vein is due to the lengthening of the right ovarian vein and the absence of competent valves [12]. In addition, dextrorotation of gravid uterus can cause compression of the right ovarian vein leading to blood stasis and therefore thrombosis [13].

In venous stasis, an infection of the ovarian venous endothelium favored by the proximity of the vagina and the uterus or an alteration of the venous wall by a direct trauma by a cesarean section or an instrumental extraction, would increase the risk of OVT [14] [15].

As the postpartum period is the preferred period for OVT, the incidence would be more increased after a caesarean section. The risk is further increased 
for cesarean sections performed for twin pregnancy [3] [7]. This is the case with our patient. The drop in blood flow, the increase in tissue trauma and bed rest after a caesarean section would be responsible for this [16].

\section{Diagnostic}

TVO has clinical signs common to other painful and febrile abdominal pathologies, and may misdiagnose and thus compromise the patient's vital prognosis. Usually the functional signs begin in the first week postpartum with diffuse abdominal pain or readily localized to the right iliac fossa, accompanied by fever up to $39^{\circ} \mathrm{C}$. They can mimic digestive pathologies with nausea, vomiting or diarrhea. Sometimes accompanied by breathing difficulties indicating a complication which is pulmonary embolism. In our case, the patient presented on the $4^{\text {th }}$ day postpartum, abdominal and pelvic pain in the right iliac fossa, and in the right flank with fever and difficulty in breathing without signs of pulmonary embolism on CT angiography.

Clinical examination can find suggestive signs in $50 \%$ of cases. There is a painful mass on abdominal palpation and the vaginal touch reveals an indurated and painful venous cord of the adnexa [17] [18]. There is a biological inflammatory syndrome with neutrophilic hyperleukocytosis and an increase in CRP. Biological samples looking for the germ are generally sterile or find E. coli infection [19]. In our reported case, the patient presented a biological inflammatory syndrome with gram-positive staphylococcus found on blood culture.

Previously diagnosed by exploratory laparotomy, today through medical imaging advances, TVO is early diagnosed. Ultrasound coupled with color Doppler would be the first examination to be performed because of its easy accessibility and safety [20]. However, the deep localization of the ovarian veins makes their ultrasound exploration difficult because of the frequent interposition of digestive gases [6]. This reduces its sensitivity and specificity by around 50\% [8]. TVO is suspected by ultrasound findings a retroperitoneal image lateral to the inferior vena cava, cylindrical, hypoechogenic. There is a slowing or even absence of flow in the vessel lumen [7].

Abdominal CT scan with contrast injection is the imaging technique of choice to search for a OVT. The thrombosed vein appears enlarged with hypodense or hyperdense content depending on the age of the thrombus, circumscribed by a peripheral border enhanced with contrast. The inflammation can result in infiltration of the fat surrounding the thrombosed vessel. Coronal reconstructions allow a better appreciation of the extension of the thrombus. On Magnetic Resonance Imaging (MRI), the same signs described on CT scan are found [6]. They have an almost identical sensitivity and specificity for the diagnosis of OVT. They are respectively $78 \%$ to $100 \%$ and $62 \%$ to $99 \%$ for abdominal and pelvic CT scan, and $92 \%$ and $100 \%$ for MRI. However, due to cost and the limited availability of MRI, CT scan is currently the examination of choice for the diagnosis of OVT [7]. It allows to specify the age and extent of the thrombus and to follow its 
evolution.

As OVT has common signs with other painful and febrile abdominal pathologies, that can lead to misdiagnosis, so it should always be considered, especially in the postpartum period, when abdominal pain with fever persists despite antibiotic treatment. This is the case reported in our observation, where despite antibiotic treatment, abdominal pain and fever persisted.

\section{Evolution and Complications}

Any diagnosis delay can lead to serious complications compromising the patient's vital prognosis. The main complication is pulmonary embolism by migration of the thrombus into the inferior vena cava, which occurs in $25 \%$ of cases with a mortality rate of $5 \%$. In addition to pulmonary embolism, patients are also at risk of sepsis [17].

When diagnosed and treated early, the evolution is often favorable with disappearance of clinical signs, biological inflammatory syndrome and repermeabilization of the ovarian vein. Recurrence of postpartum OVT in subsequent pregnancies is exceptional but remains possible [7] [17]. The prevention of postpartum OVT requires rigorous aseptic measures and the reduction of potentially traumatic gestures during delivery. A thrombophilia assessment carried out long after the episode can find congenital factors predisposing to venous thrombosis in half of the cases according to some studies [7].

\section{Traitement}

Usually the treatment for TVO is medical. It combines anticoagulants and broad-spectrum antibiotic therapy. Surgical treatment can be performed in case of contraindication to anticoagulants, or failure of medical treatment or in case of a thrombus floating in the inferior vena cava. Medical treatment consists of a combination of broad-spectrum antibiotics or antibiotics adapted to the antibiogram if a germ is found, and an anticoagulant at a curative dose. The duration of treatment is not well codified and varies between teams. In general, several teams agree to 3 - 6 months of anticoagulant and 10 days of antibiotic [9]. In our case, the patient was treated with low molecular weight heparin at a curative dose for 3 months, combined with antibiotic therapy for 14 days. The evolution has been favorable.

\section{Conclusion}

OVT is a rare pathology occurring preferentially in the postpartum period. It presents signs common to several painful abdominal pathologies which can lead to misdiagnosis. We always think about it in the postpartum period in a patient who complains of abdominal pain accompanied by fever. The diagnosis is made by imaging allowing successful medical treatment.

\section{Ethical Considerations}

We have obtained patient's consent, and reassured that information could only be used in a science-related purpose. 


\section{Conflicts of Interest}

The authors declare no conflicts of interest regarding the publication of this paper.

\section{References}

[1] Lenz, C.J., Wysokinsky, W.E., Henkin, S., Cohoon, K.P., Casanegra, A., Simmons, B.S., et al. (2017) Ovarian Vein Thrombosis: Incidence of Recurent Venous Thromboembolism and Survival. Obstetrics \& Gynecology, 130, 1127-1135. https://doi.org/10.1097/AOG.0000000000002319

[2] Basili, G., Romano, N., Bimbi, M., Lorenzetti, L., Pietrasanta, D. and Goletti, O. (2011) Postpartum Ovarian Vein Thrombosis. JSLS, 15, 286-271. https://doi.org/10.4293/108680811X13071180406673

[3] Salomon, O., Dulitzky, M. and Apter, S. (2010) New Observations in Postpartum Ovarian Vein Thrombosis: Experience of Single Centre. Blood Coagulation \& Fibrinolysis, 21, 16-19. https://doi.org/10.1097/MBC.0b013e32832f2ada

[4] Austin, O.G. (1956) Massive Thrombophlebitis of the Ovarian Vein Thrombosis. American Journal of Obstetrics and Gynecology, 72, 428-429.

https://doi.org/10.1016/0002-9378(56)90130-2

[5] Kominiarek, M.A. and Hibbard, J.U. (2006) Postpartum Ovarian Vein Thrombosis: An Update. Obstetrical \& Gynecological Survey, 61, 337-342.

https://doi.org/10.1097/01.ogx.0000216564.53044.f1

[6] Meuwly, J.Y, Kawkabani-Marchini, A. and Sgourdos, G. (2012) Thrombose Veineuse Ovarienne. Forum Médical Suisse, 12, 144-148. https://doi.org/10.4414/fms.2012.07784

[7] Lerouge, J., Sanguin, S., Gondry, J. and Sergent, F. (2016) Prise en charge de la thrombose veineuse ovarienne du post-partum. L'expérience du CHU d'Amiens. Gynécologie Obstétrique \& Fertilité, 44, 88-95. https://doi.org/10.1016/j.gyobfe.2015.11.010

[8] Togan, T., Turan, H., Cifci, E. and Çiftci, C. (2015) Ovarian and Renal Vein Thrombosis: A Rare Cause of Fever Outer the Postpartum Period. Case Reports in Obstetrics and Gynecology, 2015, Article ID: 817862. https://doi.org/10.1155/2015/817862

[9] Arkadopoulos, N., Dellaportas, D., Yiallourou, A., Koureas, A. and Voros, D. (2011) Ovarian Vein Thrombosis Mimicking Acute Abdomen: A Case Report and Literature Review. World Journal of Emergency Surgery, 6, Article No. 45. https://doi.org/10.1186/1749-7922-6-45

[10] Salomon, O., Apter, S., Shaham, D., Hiller, N., Bar-Ziv, J., Itzchak, Y., et al. (1999) Risk Factors Associated with Postpartum Ovarian Vein Thrombosis. Thrombosis and Haemostasis, 82, 1015-1019. https://doi.org/10.1055/s-0037-1614321

[11] Calderwood, C.J., Jamieson, R. and Greer, I.A. (2007) Gestational Related Changes in the Deep Venous System of the Lower Limb on Light Reflection Rheography in Pregnancy and the Puerperium. Clinical Radiology, 62, 1174-1179. https://doi.org/10.1016/j.crad.2007.06.003

[12] Harris, K., Mehta, S. and Iskhakovetal, E. (2012) Ovarian Veinthrombosis in the Non Pregnant Woman: An Over Looked Diagnosis. Therapeutic Advances in Hematology, 3, 325-328. https://doi.org/10.1177/2040620712450887

[13] Narayanmoorthy, S., Ganesan, P. and Ramanan, R. (2015) A Rare Case of Postpartum Ovarian Vein Thrombosis. International Journal of Reproduction, Contracep- 
tion, Obstetrics and Gynecology, 4, 878-880.

https://doi.org/10.18203/2320-1770.ijrcog20150117

[14] Maldjian, P.D. and Zurlow, J. (1997) Ovarian Vein Thrombosis Associated with a Tubo-Ovarian Abscess. Archives of Gynecology and Obstetrics, 261, 55-58. https://doi.org/10.1007/s004040050199

[15] Quarello, E., Desbriere, R., Hartung, O., Portier, F., d'Ercole, C. and Boubli, L. (2004) Thrombophlebite de la veine ovarienne du post-partum. Journal de Gynécologie Obstétrique et Biologie de la Reproduction, 33, 430-440. https://doi.org/10.1016/S0368-2315(04)96551-9

[16] Cao, W., Ni, X., Wang, Q., Li, J., Li, Y., Chen, T., et al. (2020) Early Diagnosis and Precision Treatment of Right Ovarian Vein and Inferior Vena Cava Thrombosis Following Caesarean Section: A Case Report. Experimental and Therapeutic Medicine, 19, 2923-2926. https://doi.org/10.3892/etm.2020.8548

[17] Dougan, C., Phillips, R., Harley, I., Benson, G. and Anbazhagan, A. (2016) Postpartum Ovarian Vein Thrombosis. The Obstetrician \& Gynaecologist, 18, 291-299. https://doi.org/10.1111/tog.12295

[18] Jenayah, A.A., Saoudil, S., Boudaya, F., Bouriel, I., Sfar, E. and Chelli, D. (2015) Ovarian Vein Thrombosis. Pan African Medical Journal, 21, Article No. 251. https://doi.org/10.11604/pamj.2015.21.251.6908

[19] Simons, G.R., Piwnica-Worms, D.R. and Goldhaber, S.Z. (1993) Ovarian Vein Thrombosis. American Heart Journal, 126, 641-647.

https://doi.org/10.1016/0002-8703(93)90415-6

[20] Witlin, A. (1995) Postpartum Ovarian Vein Thrombosis after Vaginal Delivery: A Report of 11 Cases. Obstetrics \& Gynecology, 85, 775-780.

https://doi.org/10.1016/0029-7844(95)00040-X 